\title{
A lesion-derived brain network for emotion regulation
}

Jing Jiang ( $\nabla_{\text {jjiang17@bwh.harvard.edu })}$

Brigham and Women's Hospital

Michael Ferguson

Harvard Medical School

Jordan Grafman

Shirley Ryan AbilityLab

Alexander Cohen

Boston Children's Hospital https://orcid.org/0000-0001-6557-5866

Michael Fox

Harvard University

Article

Keywords: Emotion regulation, functional connectivity, brain lesion, lesion network mapping, fMRI

Posted Date: February 24th, 2022

DOI: https://doi.org/10.21203/rs.3.rs-1363458/v1

License: (c) (i) This work is licensed under a Creative Commons Attribution 4.0 International License.

Read Full License 


\section{Title: A lesion-derived brain network for emotion regulation}

\section{Authors:}

Jing Jiang ${ }^{1,2, *}$, Michael A. Ferguson ${ }^{1,2}$, Jordan Grafman ${ }^{4,5}$, Alexander L. Cohen ${ }^{1,2,3, \#}$ and Michael D. Fox ${ }^{1,2, \#}$

\section{Affiliations:}

1. Center for Brain Circuit Therapeutics, Brigham \& Women's Hospital, Boston, MA, USA

2. Department of Neurology, Harvard Medical School, Boston, MA, USA

3. Department of Neurology, Boston Children's Hospital, Boston, MA, USA

4. Feinberg School of Medicine, Northwestern University, Chicago, IL, USA

5. Shirley Ryan Ability Lab, Chicago, IL, USA

* Corresponding author

Jing Jiang, Ph.D.

Center for Brain Circuit Therapeutics,

Brigham \& Women's Hospital | Harvard Medical School

75 Francis St, Boston, MA 02115

jiiang17@,bwh.harvard.edu

\# Contributed equally to this work

\section{Key Words}

Emotion regulation, functional connectivity, brain lesion, lesion network mapping, fMRI

\section{Author contributions}

J.J., A.L.C., and M.D.F conceptualized and developed methods, J.J. A.L.C., and M.A.F performed data analyses. J.J wrote manuscript drafts. J.G. provided lesions with behavioral and demographic data. All authors revised manuscript. 


\begin{abstract}
Emotion regulation has been linked to specific brain networks based on functional neuroimaging. We found that damage to these networks was associated with emotion regulation impairment in patients following focal brain injury $(n=167)$. Next, we used this lesion dataset to derive a de novo brain network for emotion regulation, which was defined by functional connectivity to the left ventrolateral prefrontal cortex (vlPFC). Finally, we used an independent lesion database ( $n=$ $629)$ to test whether damage to this lesion-derived network would increase the risk of neuropsychiatric conditions associated with emotion regulation impairment. We found that lesions causing mania, criminality, and depression intersected this network more than lesions causing other disorders. We conclude that emotion regulation maps to a connected brain network centered on the left vlPFC. Damage to this network impairs emotion regulation and may increase the risk of specific neuropsychiatric disorders.
\end{abstract}




\section{Introduction}

2 Emotion regulation refers to the ability to effectively manage our own and others' emotional states ${ }^{1,2}$. Effective emotion regulation is closely associated with increased quality of interpersonal relationships and well-being ${ }^{3,4}$. Conversely, impaired emotion regulation is regarded as a transdiagnostic risk factor for several psychiatric disorders, such as depression, anxiety, autism, and bipolar disorder ${ }^{5-9}$. As such, understanding the neural substrates of emotion regulation may provide insight into biomarker development and treatment strategies and has been a topic of intense investigation over the last two decades ${ }^{10}$.

Functional neuroimaging ${ }^{11-15}$ and computational neural models e.g., ${ }^{6,16-20}$ have implicated The most recent and comprehensive meta-analysis of these functional neuroimaging results identified a set of brain networks associated with emotion regulation (Fig. 1), which were distinct from networks associated with emotion perception and generation ${ }^{10}$. However, this work is solely based on task-related observational data in healthy adults, which are correlational in nature. As such, it remains unknown whether these networks are causally involved in emotion regulation.

Patients with focal brain lesions can provide unique insight into the causal dependencies between mental functions and neural substrates in the human brain ${ }^{21-23}$. Lesions in multiple different brain locations have been associated with changes in emotion regulation including the left dorsolateral and dorsomedial PFC (dlPFC and dmPFC) ${ }^{24}$, the ventromedial PFC / ventral anterior cingulate cortex ( $\mathrm{vmPFC} / \mathrm{VACC})^{25-27}$, right vlPFC ${ }^{28}$, and right superior frontal gyrus (SFG) ${ }^{29}$. It is now possible to test whether lesions in different brain locations that cause a common symptom map to a connected brain network ${ }^{22,30}$. To our knowledge, this lesion network mapping approach has not been applied to emotion regulation.

In the present study, we first test whether lesions to a priori networks derived from functional neuroimaging ${ }^{10}$ are associated with emotion regulation impairment in a Vietnam dataset $(n=$ 167 ), and whether this impairment is specific to emotion regulation versus other components of emotion processing. Second, we leverage lesion locations associated with emotion regulation impairment along with the human brain connectome to derive an empirical / de novo network for emotion regulation. Finally, we test whether damage to this lesion-derived emotion regulation network increase the risk of neuropsychiatric conditions associated with emotion regulation impairment in an independent lesion database $(n=629)$.

\section{Methods}

\section{A priori networks for emotion regulation from functional neuroimaging}

Two emotion regulation networks were identified in Morawetz, et al. ${ }^{10}$ and are used as a priori networks in the present study (Fig. 1). The first network (here referred to as the "Dorsal Network") was most associated with response inhibition and executive control elements of emotion regulation, and included regions in the bilateral dlPFC, bilateral inferior parietal cortex (IPL), SFG, and supplementary motor area. The second network (here referred to as the "Ventral Network") was most associated with reappraisal and language processing elements of emotion regulation and included regions in the bilateral vlPFC, a.k.a., inferior frontal gyrus (IFG), left middle temporal gyrus/superior temporal sulcus (MTG/STS), and left posterior STS/temporoparietal junction (TPJ). We combined these two networks into a single a priori 
emotion regulation network for our main data analyses, but also performed analyses on each network individually.

\section{Lesion Locations}

Patients $(n=167$, mean age $58.1 \pm 2.8$ years, all men) were drawn from the Phase 3 registry of the prospective W.F. Caveness Vietnam Head Injury Study ${ }^{31,32}$. These patients were Vietnam War veterans who suffered brain damage from penetrating head injuries during combat, as described in previous studies ${ }^{33,34}$. The present study was approved by the Institutional Review Board at Brigham and Women's Hospital (2020P002987). Lesion locations for each patient were traced from chronic neuroimaging and transformed to MNI atlas space, as documented in prior work $^{33,34}$.

\section{Behavioral measurements in lesion patients}

Emotion regulation ability. The emotion regulation ability of each patient was assessed with the "Managing emotions" subscale of the Mayer-Salovey-Caruso Emotional Intelligence Test (MSCEIT) (Version 2.0) ${ }^{2}$. MSCEIT is a valid standardized ability-measure of emotional intelligence behaviors ${ }^{35}$, which is conceptually and empirically distinct from self-report questionnaire measurements of emotional intelligence such as the Emotional-Quotient Inventory 36. The "Managing emotion" subscale is one of four branches of emotional intelligence measured by this test and assesses the ability to modulate mood and emotions in oneself and other people. It includes 29 items that require participants to answer how effective different strategies are for dealing with emotion-laden intrapersonal and interpersonal situations depicted in short stories. For example, participants are asked to rate the effectiveness (from "Very ineffective" to "Very effective") of a strategy/action that may help another person maintain a peaceful mood, reduce sad feelings, or enhance a joyful mood. Higher scores on this subscale represent stronger emotion regulation ability.

Other emotion processing abilities. Three other emotion processing abilities were assessed with additional subscales of the MSCEIT ${ }^{2}$, i.e., "Perceiving emotions", "Using emotions", and "Understanding emotions". The "Perceiving emotions" subscale measures the ability to perceive emotions in oneself and others as well as in objects, art, stories, music, and other stimuli. The "Using emotion" subscale measures the ability to generate, feel, and use emotions to facilitate thinking. The "Understanding emotion" subscale measures the ability to understand complex emotions, how emotions combine, progress, and transition from one to the other. Likewise, higher scores on these subscales represent stronger abilities.

82

Verbal ability. The verbal language ability of each patient was assessed with the Verbal Comprehension Index Scale of the Wechsler Adult Intelligence Scale-III (WAIS-III) ${ }^{37}$. This subscale provides a valid standardized ability-measure of abstract verbal reasoning, oral expression, and acquired verbal knowledge from culture, social conventions, etc. For example, participants are asked to orally answer questions such as: "In what way are an apple and a pear alike?", "What is a guitar?", and "What do your lungs do?". Higher scores on this scale represent

89 stronger language ability.

90

\section{Relationship between lesion locations and emotion regulation ability}


Each patient's lesion location was tested for voxel-wise intersection with an overall a priori mask including both the Dorsal Network and Ventral Network. Patients with lesions intersecting with this a priori mask were defined as the "Hit" group, while those without intersection were defined as the "No-Hit" group. Each patient's lesion location was also tested for voxel-wise intersection with the Dorsal Network and Ventral Network separately.

97

To test whether lesions to the overall a priori networks were associated with emotion regulation ability, we compared the emotion regulation scores in patients of the Hit group and the No-Hit group with Welch's unequal variances $t$-test (an adaption of Student's $t$-test for unequal sample sizes in two groups). We next quantified damage as a continuous variable, i.e., percentage of the overall a priori networks that was damaged by a particular patient's lesion. We then used linear regression to test for relationships between network damage percentages and emotion regulation scores. The distributions of individual network damage percentages are shown in Fig. S1.

To further examine whether either a priori network plays a more central role in emotion regulation, we compared the emotion regulation scores between two lesion groups for the Dorsal 108 Network and the Ventral Network separately with Welch's $t$-test. We also performed a multiple linear regression relating emotion regulation scores to the network damage percentages of both the Dorsal and Ventral Network.

\section{Lesion network mapping}

113 We used a recently validated method, lesion network mapping 22,30,38,39 to derive an empirical network of regions consistently associated with impairment of emotion regulation. First, we computed the typical/normative resting-state functional connectivity (RSFC) between each lesion location and the rest of the brain using a publicly available connectome dataset of 1000 healthy participants (18-35 years old, mean age 21.3 years, $42.7 \%$ males) from the Genome Superstruct Project (GSP) ${ }^{40,41}$. This generated a map of brain regions functionally connected to each patient's lesion location, i.e., a "lesion connectivity map". Next, we computed voxel-wise general linear models comparing unthresholded connectivity maps to emotion regulation scores using the Permutation Analysis of Linear Models (PALM) software (1000 permutations), including lesion size as a covariate. Regions associated with emotion regulation were identified by voxel-wise False Discovery Rate (FDR) $(p<0.05)$ correction within the a priori mask (cluster size $k>10$ ). If multiple regions were identified, a stepwise linear regression model was performed to determine the predominant predictor(s), referred to as "hub region(s)", for emotion regulation. We also repeated this PALM analysis across all grey matter voxels (cortical and subcortical) to confirm our findings at the whole-brain level.

\section{Defining a lesion-derived brain network for emotion regulation}

The above analysis identified the left vlPFC as a hub region which was used as a seed ROI to compute a brain map of regions that, if lesioned, would be associated with emotion regulation ability. This would include our original set of lesions associated with emotion regulation impairment but may also include additional regions not seen in our specific cohort. This approach has been previously validated to define brain networks for memory ${ }^{38}$, depression ${ }^{42}$, and prosopagnosia ${ }^{39}$.

\section{Network damage score}


We next computed a "network damage score" for each patient by summing the functional connectivity values at lesion locations within the unthresholded lesion-derived brain network for emotion regulation noted above. Lesion size was regressed from the network damage score and the residual used as an adjusted network damage score, used in all analyses reported in this study.

\section{Relationship to lesions causing neuropsychiatric disorders}

Finally, we tested whether damage to our lesion-derived emotion regulation network would dissociate neuropsychiatric disorders previously associated with impaired emotion regulation from those without. To do so, we computed network damage scores as described above for 629 additional patients with lesions associated with a range of disorders from 17 independent lesion datasets. These disorders represent all lesion network mapping studies previously published by our laboratory at the time of manuscript preparation. Specific neuropsychiatric disorders associated with impaired emotion regulation ${ }^{5-9}$ included mania $(n=56)^{43}$, criminality $(n=40)$ ${ }^{44}$, and depression $(n=36)^{42}$. Patients with non-mood disorders $(n=497)$ were categorized together as "other disorders". A one-way analysis of variance (ANOVA) on network damage scores across these 4 categories was performed to test for any relationship between neuropsychiatric disorders caused by brain lesions and our lesion-derived emotion regulation network. This ANOVA was repeated without combining "other disorders" - 17 diagnoses studied with lesion network mapping - from separate datasets to test the generalizability of our findings. We also repeated this analysis using the original a priori network(s) to compare the performance of our novel lesion-derived network.

\section{Results}

\section{Lesion locations and intersection with the a priori networks}

Lesion locations in the Vietnam dataset covered most cortical and subcortical areas, including the a priori brain networks from Morawetz, et al. ${ }^{10}$ (Fig. 2A). Lesions from 139 patients (83.2\%) "hit" the overall a priori networks (Hit group), while 28 (16.8\%) did not (No-Hit group) (Fig. 2B, Fig. S1-2). Lesions from 112 patients $(67.1 \%)$ hit the Dorsal Network, while lesions from 95 patients $(56.9 \%)$ hit the Ventral Network (Fig. S1-2).

\section{Damage to the overall a priori networks are associated with emotion regulation impairment} Emotion regulation scores were significantly lower in the Hit group $(M=89.4, S D=11.2)$ than for the No-Hit group $(M=94.2, S D=9.9)$ using the overall a priori network (Welch's $t_{(42)}=$ $2.28, p=0.028)$ (Fig. 3A), indicating decreased emotion regulation ability is associated with lesions intersecting the overall a priori networks. This relationship held when brain lesions were quantified as a continuous variable, i.e., network damage percentage. Higher network damage was correlated with greater emotion regulation impairment $\left(\mathrm{Beta}=-0.64, t_{(165)}=-3.33, p=\right.$ 0.001) (Fig. 3B). This effect remained significant after controlling for lesion size (Beta $=-0.68, t$ $(164)=-2.25, p=0.025)$. This effect also remained significant when patients with no lesions to the overall a priori networks were excluded (Beta $=-0.56, t_{(164)}=-2.73, p=0.007$ ).

\section{Damage to the Ventral rather than the Dorsal Network is associated with emotion regulation impairment}

After dividing the overall a priori networks into Dorsal and Ventral components, as originally identified by Morawetz, et al. ${ }^{10}$, we found that emotion regulation scores did not differ 
significantly between patients with or without lesions hitting the Dorsal Network (Welch's $t_{\text {(117) }}$ $=-1.34, p=0.182)($ Fig. 3C). However, emotion regulation scores were significantly lower in patients with lesions hitting the Ventral Network compared to those without (Ventral Hit: $M=$ 87.8, $S D=11.2$ vs. No-Hit: $M=93.2, S D=10.4$; Welch's $\left.t_{(158)}=-3.22, p=0.002\right)$ (Fig. 3E). Multiple linear regression analysis revealed that this relationship also held when brain lesion damage to the networks was quantified as a continuous variable. That is, emotion regulation scores were not significantly associated with network damage percentage of the Dorsal Network $\left(\right.$ Beta $\left.=-0.00, t_{(164)}=-0.02, p=0.982\right)($ Fig. 3D), but were significantly associated with network damage percentage of the Ventral Network (Beta $\left.=-0.69, t_{(164)}=-3.69, p<0.001\right)($ Fig. 3F). As with lesions to the overall a priori networks, this effect remained significant after controlling for lesion size $\left(\right.$ Beta $\left.=-0.67, t_{(163)}=-3.11, p=0.002\right)$.

\section{Damage to the Ventral Network is specific for emotion regulation impairment}

To test whether the Ventral Network is specific to emotion regulation or involved in general emotional processing, we examined the effect of lesions to the Ventral Network on three other emotion processing scores ("Perceiving emotions", "Using emotion", and "Understanding emotion"). Damage to the Ventral Network was correlated with all three emotion processing scores (path c in Fig. 4). However, these scores are all tightly correlated with one another and with emotion regulation scores ${ }^{45}$. To test which scores were most associated with Ventral Network damage (controlling for other scores), we performed multiple mediation analyses. We found that damage to the Ventral Network was no longer associated with any other emotional processing scores after controlling for emotion regulation (path c' in Fig. 4). In contrast, damage to the Ventral Network remained correlated with emotion regulation after controlling for any of the other emotional processing scores (path c' in Fig. S3A-C).

Since the Ventral Network largely overlaps with regions implicated in language processing, we also performed a mediation analysis for Verbal ability scores. As above, we found that damage to the Ventral Network was no longer associated with Verbal ability scores after controlling for emotion regulation (path c' in Fig. S3D). While damage to the Ventral Network remained correlated with emotion regulation after controlling for Verbal ability scores (path c' in Fig. S3E).

\section{Lesion network mapping implicates connections with left vIPFC in emotion regulation}

While lesions to the Ventral Network are more likely to cause emotion regulation impairment, the topography of this network could be refined by deriving a de novo emotion regulation network based on lesion-induced impairments. Using lesion network mapping to derive this network in a data-driven manner (Fig. 5A-B), we found that connectivity between lesion locations and specific brain regions within the overall a priori networks was correlated with emotion regulation scores. These regions included the left vlPFC, left STS and left TPJ (FDR $p$ $<0.05, k>10)$ (Fig. 5C). We further used stepwise linear regression to determine whether any of these three regions was more tightly linked to emotion regulation. This highlighted that connectivity between lesion locations and the left vlPFC (Beta $=-0.13, t=3.36, p<0.001)$ was the strongest correlate of emotion regulation impairment. Repeating this analysis at the wholebrain level found the most significant findings were indeed in the left vlPFC (Fig. S4). 
Connectivity with the left vIPFC defines a network of brain regions potentially associated 230 with emotion regulation By definition, functional connectivity (FC) with the left vlPFC defines a brain network that encompasses lesion locations associated with emotion regulation impairment (Fig. 6A). As expected, network damage scores on the vlPFC-seeded FC map were significantly associated with emotion regulation impairment (Beta $\left.=-3.7 \mathrm{e}-05, t_{(164)}=-3.27, p=0.001\right)$ (Fig. 6B-C), i.e., patients with more damage to this map were more likely to have emotion regulation impairment. The topography of this lesion-derived network is similar to the a priori Ventral Network (green outlines in Fig. 6A), but it is not identical. In fact, repeating our linear regression analysis after excluding all voxels within the Ventral Network (Fig. S5), still results in a significant association between damage to our lesion-derived network and emotion regulation (Beta $=-1.7 \mathrm{e}-05, t_{(164)}=$ $-3.01, p=0.003)($ Fig. S5).

\section{Damage to the lesion-derived network for emotion regulation is relevant in other lesion- induced neuropsychiatric disorders}

Finally, using 629 lesions causing diverse disorders, we found that lesions associated with impaired emotion regulation intersected our network more than other lesions (one-way ANOVA, $\left.\mathrm{F}_{(3)}=6.98, p=0.0001\right)$ (Fig. 7). Specifically, lesions causing mania $\left(t_{(551)}=3.58, p=0.0004\right)$, criminality $\left(t_{(535)}=2.88, p=0.004\right)$, and depression $\left(t_{(531)}=2.31, p=0.02\right)$, showed significantly higher network damage scores than other disorders. The pattern was similar when comparing across all 17 disorders, with no assumptions as to which disorders should be associated with emotion regulation (one-way ANOVA, $\left.\mathrm{F}_{(16)}=2.20, p=0.005\right)$ (Fig. S6). In contrast, repeating our analyses using the a priori Ventral Network from the task-fMRI literature did not show a significant effect across different neuropsychiatric disorders (one-way ANOVA, $\left.\mathrm{F}_{(16)}=1.14, p=0.31\right)($ Fig. S6).

\section{Discussion}

Here, we show that damage to an a priori emotion regulation network defined by task-fMRI is associated with emotion regulation impairment in patients with brain lesions. We then derive a de novo brain network for emotion regulation based on these lesion locations. This lesionderived emotion regulation network is defined by connectivity to the left vlPFC. Finally, we show that lesions causing mania, criminality, and depression impact this lesion-derived emotion regulation network more often than other lesion-induced neuropsychiatric conditions across multiple independent datasets.

Many brain regions have been implicated in emotion regulation based on task-based fMRI including the a priori dorsal and ventral emotion regulation networks used here ${ }^{10}$, as well as many regions within in these networks including the dlPFC, vlPFC, and TPJ e.g., ${ }^{6,11-20}$. While valuable, imaging correlates of task involvement are limited in several important ways: they do not support causal inferences, nor do they imply that any specific region is necessary and sufficient for the emotion regulation process.

Our work provides lesion-based validation of these prior fMRI results, showing that damage to these networks impairs emotion regulation. Moreover, we found that lesions hitting the ventral $a$ priori emotion regulation network, which is left-lateralized, are associated with more severe 
emotion regulation impairment as compared to hitting the dorsal a priori emotion regulation network, which includes bilateral prefrontal and parietal areas.

Brain lesions causing emotion regulation impairment have been reported in many different brain locations including left dlPFC and dmPFC ${ }^{24}$, vmPFC/vACC ${ }^{25-27}$, right vlPFC ${ }^{28}$, and right SFG 29 . Our results help reconcile this heterogeneity, as these locations are all within our lesionderived network for emotion regulation. In other words, lesions in multiple different brain locations can lead to impaired emotion regulation, but these lesion locations all fall within a specific brain network. This result is consistent with lesion network mapping results across a variety of other neurological and psychiatric disorders $30,39,42,43,46$.

The lesion-derived network for emotion regulation identified here, i.e., regions with positive connectivity with the left vlPFC, aligns well with the ventral component of the task-fMRI derived a priori emotion regulation networks ${ }^{10}$. Our work is consistent with, but expands upon, prior work using a subset of this same dataset and voxel-based lesion symptom mapping ${ }^{47}$ to explore the neural bases of facets of emotional intelligence ${ }^{48,49}$. Our finding also suggests that the larger the impact on this vlPFC-centered network, the more severe the impairment in emotion regulation.

The vlPFC has been implicated in the language processing e.g., ${ }^{50}$, inhibitory control ${ }^{51}$, and task-set switching ${ }^{52}$. These functions may share a "regulation" process or strategy such as reappraisal, suppression, and distraction that lead to the vlPFC to be involved in these cognitive processes ${ }^{53,54}$. Surprisingly, we are not aware of any documented cases of a focal lesion to the left vlPFC causing emotion regulation impairment; however, lesions or stimulations to the homologous right vIPFC has been shown to impair or improve emotion regulation ability in a few studies ${ }^{28,55}$. This may represent a case of diaschisis ${ }^{56}$, where lesions or stimulation to the right vlPFC influence emotion regulation ability through modulated connectivity to the left vlPFC, while damage to the left vlPFC itself may cause a much broader phenotype with more severe deficits, obviating a specific detectable deficient in emotion regulation. Whether the left vlPFC mediates the relation between the right vlPFC and emotion regulation is a testable hypothesis for future work.

Nonetheless, the topography of our lesion-derived network for emotion regulation is not equivalent to either the ventral a priori emotion regulation network or networks identified in other prior meta-analytic studies. As shown above, we demonstrate a strong statistical relationship between emotion regulation ability and lesion connectivity, even when the entire ventral a priori network is excluded (Fig. S5). Regions with strong positive connectivity to the left vIPFC include the vmPFC, SFG, left anterior temporal cortex, right pSTS, posterior cingulate cortex, and bilateral cerebellar regions (posterior Crus 1 and 2) (Fig. S5). This last connection is intriguing as a role for the cerebellum in emotion regulation has long been postulated ${ }^{57}$ and has gained popularity since the 1970s. Important from a clinical standpoint, the specific cerebellar region (posterior Crus 1 and 2) included in our lesion-derived network (posterior Crus 1 and 2) is more easily accessible than that in the ventral a priori emotion regulation network (anterior Crus 1) for non-invasive stimulation ${ }^{58,59}$. 
We also here demonstrate the importance of vlPFC connectivity for emotional regulation in other lesion-induced neuropsychiatric disorders. Lesions causing mania, criminality, and depression disorders associated with impaired emotional regulation - were more likely to impact our lesionderived emotion regulation network than lesions associated with disorders not associated with emotional regulation impairment. This is consistent with prior work suggesting that emotion regulation impairment may serve as a transdiagnostic risk factor ${ }^{5-8,60,61}$ for a variety of neuropsychiatric disorders, including those studied here, e.g., mania ${ }^{62}$, criminal behavior ${ }^{63}$, and depression ${ }^{64,65}$. Moreover, the "hub regions" for these disorders identified in our prior lesion network mapping work also fall within our lesion-derived network for emotion regulation: right orbital PFC for mania ${ }^{43}$, inferior orbital PFC and anterior temporal lobes for criminal behavior ${ }^{44}$, and the left dlPFC for depression ${ }^{42}$.

Unexpectedly, we found that lesions associated with prosopagnosia intersected negative nodes of our lesion-based emotion regulation network. Although speculative, this might suggest that lesions causing prosopagnosia would be associated with intact or even improved emotion regulation. This unexpected result is consistent with a recent study showing poor face recognition performance associated with a higher level of emotion regulation ${ }^{66}$.

Several limitations in this study should be mentioned here. First, our lesion cases were all male veterans who suffered penetrating traumatic brain injuries during the Vietnam War. As such, our findings may not generalize to people with other genders, ages, and backgrounds ${ }^{67}$. Second, the MSCEIT focuses on the reappraisal aspect of emotion regulation. While other constructs include strategies such as distraction and suppression which may localize differently ${ }^{53,54}$, findings from a recent meta-analysis study help mitigate this concern. They found that the left vlPFC, the bilateral anterior insula, and the SMA, which are included in our network, were consistently involved in emotion regulation independent of strategies and were not specific for any specific strategy ${ }^{15}$. Third, we used a normative connectome dataset to approximate the connectivity pattern for each patient at the time their lesion occurred. This increases signal-to-noise to estimate connectivity but ignores individual variations that may be important to account for emotion regulation change. While having RSFC data from the patients themselves would alleviate this concern, it is typically not available - as is the case here.

In conclusion, we identified a specific brain network for emotion regulation, centered on the left vlPFC, from a large dataset of focal brain lesions. This network helps validate recent task-fMRI findings and shows generalizability to independent lesion datasets associated with disorders of emotion regulation. 


\section{Acknowledgments}

357 This work was supported by a BBRF Young Investigator Grant 29441 to J.J., M.D.F. was

358 supported by the Nancy Lurie Marks Foundation, the Kaye Family Research Endowment, the

359 Ellison / Baszucki Foundation, and the NIH (R01MH113929, R21MH126271, R56AG069086,

360 R01MH1 15949, and R01AG060987), A.C. was supported by a Shields Research grant from the

361 Child Neurology Foundation (AC) and by the NIH/NIMH K23MH120510.

362

363 Competing Interests

364 The authors declare no competing interests. 


\section{References}

3661 Gross, J. J. Emotion regulation in adulthood: Timing is everything. Current directions in

367 psychological science 10, 214-219 (2001).

3682 Mayer, J. D., Salovey, P., Caruso, D. R. \& Sitarenios, G. Measuring emotional

369 intelligence with the MSCEIT V2. 0. Emotion 3, 97 (2003).

3703 Gross, J. J. \& John, O. P. Individual differences in two emotion regulation processes:

371 implications for affect, relationships, and well-being. Journal of personality and social

$372 \quad$ psychology 85, 348 (2003).

3734 Berking, M. \& Wupperman, P. Emotion regulation and mental health: recent findings, current challenges, and future directions. Current opinion in psychiatry 25, 128-134 (2012).

5 Etkin, A. \& Schatzberg, A. F. Common abnormalities and disorder-specific compensation during implicit regulation of emotional processing in generalized anxiety and major depressive disorders. American Journal of Psychiatry 168, 968-978 (2011).

6 Phillips, M. L., Ladouceur, C. D. \& Drevets, W. C. A neural model of voluntary and automatic emotion regulation: implications for understanding the pathophysiology and neurodevelopment of bipolar disorder. Molecular psychiatry 13, 833-857 (2008). Mazefsky, C. A. et al. The role of emotion regulation in autism spectrum disorder. Journal of the American Academy of Child \& Adolescent Psychiatry 52, 679-688 (2013). 8 Sloan, E. et al. Emotion regulation as a transdiagnostic treatment construct across anxiety, depression, substance, eating and borderline personality disorders: A systematic review. Clinical psychology review 57, 141-163 (2017).

9 Bufkin, J. L. \& Luttrell, V. R. Neuroimaging studies of aggressive and violent behavior: current findings and implications for criminology and criminal justice. Trauma, Violence, \& Abuse 6, 176-191 (2005).

10 Morawetz, C. et al. Multiple large-scale neural networks underlying emotion regulation. Neuroscience \& Biobehavioral Reviews (2020).

11 Buhle, J. T. et al. Cognitive reappraisal of emotion: a meta-analysis of human neuroimaging studies. Cerebral cortex 24, 2981-2990 (2014).

12 Frank, D. et al. Emotion regulation: quantitative meta-analysis of functional activation and deactivation. Neuroscience \& Biobehavioral Reviews 45, 202-211 (2014).

13 Kohn, N. et al. Neural network of cognitive emotion regulation - an ALE meta-analysis and MACM analysis. Neuroimage 87, 345-355 (2014).

14 Messina, I., Bianco, S., Sambin, M. \& Viviani, R. Executive and semantic processes in reappraisal of negative stimuli: insights from a meta-analysis of neuroimaging studies.

Frontiers in Psychology 6, 956 (2015).

409 Morawetz, C., Bode, S., Derntl, B. \& Heekeren, H. R. The effect of strategies, goals and stimulus material on the neural mechanisms of emotion regulation: a meta-analysis of fMRI studies. Neuroscience \& Biobehavioral Reviews 72, 111-128 (2017).

6 Smith, R. \& Lane, R. D. The neural basis of one's own conscious and unconscious emotional states. Neuroscience \& Biobehavioral Reviews 57, 1-29 (2015).

7 Etkin, A., Büchel, C. \& Gross, J. J. The neural bases of emotion regulation. Nature reviews neuroscience 16, 693-700 (2015).

18 Silvers, J. A. \& Moreira, J. F. G. Capacity and tendency: A neuroscientific framework for the study of emotion regulation. Neuroscience letters 693, 35-39 (2019). 
41019 Dixon, M. L., Thiruchselvam, R., Todd, R. \& Christoff, K. Emotion and the prefrontal cortex: An integrative review. Psychological bulletin 143, 1033 (2017). Ochsner, K. N., Silvers, J. A. \& Buhle, J. T. Functional imaging studies of emotion regulation: a synthetic review and evolving model of the cognitive control of emotion. Annals of the new York Academy of Sciences 1251, E1 (2012). Adolphs, R. Human lesion studies in the 21st century. Neuron 90, 1151-1153 (2016). Fox, M. D. Mapping Symptoms to Brain Networks with the Human Connectome. N Engl J Med 379, 2237-2245, doi:10.1056/NEJMra1706158 (2018). Siddiqi, S., K, K., J, P. \& MD, F. Causal Mapping of Human Brain Function Nature Reviews Neuroscience In Press (2021).

24 Salas, C. E. et al. "Opening an emotional dimension in me": Changes in emotional parietal damage. Bulletin of the Menninger Clinic 78, 301-334 (2014).

25 Maier, M. E. \& Di Pellegrino, G. Impaired conflict adaptation in an emotional task context following rostral anterior cingulate cortex lesions in humans. Journal of cognitive neuroscience 24, 2070-2079 (2012).

26 Motzkin, J. C., Philippi, C. L., Wolf, R. C., Baskaya, M. K. \& Koenigs, M. Ventromedial prefrontal cortex is critical for the regulation of amygdala activity in humans. Biological psychiatry 77, 276-284 (2015). the bed nucleus of stria terminalis. cortex 64, 281-288 (2015).

28 Salas, C. E. et al. 'Just can't hide it': a behavioral and lesion study on emotional response modulation after right prefrontal damage. Social Cognitive and Affective Neuroscience 11, 1528-1540 (2016).

29 Falquez, R. et al. Detaching from the negative by reappraisal: the role of right superior frontal gyrus (BA9/32). Frontiers in Behavioral Neuroscience 8, 165 (2014).

30 Boes, A. D. et al. Network localization of neurological symptoms from focal brain lesions. Brain 138, 3061-3075, doi:10.1093/brain/awv228 (2015).

31 Caveness, W. \& WF, C. Incidence of craniocerebral trauma in the United States in 1976 with trend from 1970 to 1975. (1979).

32 Raymont, V., Salazar, A., Krueger, F. \& Grafman, J. "Studying injured minds"-the Vietnam head injury study and 40 years of brain injury research. Frontiers in Neurology 2, 15 (2011).

33 Krueger, F. et al. The neural bases of key competencies of emotional intelligence. Proceedings of the National Academy of Sciences 106, 22486-22491 (2009).

34 Barbey, A. K. et al. Lesion mapping of social problem solving. Brain 137, 2823-2833 (2014).

35 Mayer, J. D., Roberts, R. D. \& Barsade, S. G. Human abilities: Emotional intelligence. Annu. Rev. Psychol. 59, 507-536 (2008).

36 Bar-On, R. The BarOn emotional quotient inventory (BarOn EQ-i). Toronto, ON: MultiHealth Systems Inc (1997).

37 Wechsler, D. (APPENDIXES, 1997).

38 Ferguson, M. A. et al. A human memory circuit derived from brain lesions causing amnesia. Nat Commun 10, 3497, doi:10.1038/s41467-019-11353-z (2019).

39 Cohen, A. L. et al. Looking beyond the face area: lesion network mapping of prosopagnosia. Brain 142, 3975-3990, doi:10.1093/brain/awz332 (2019). 
$45640 \quad$ Yeo, B. T. et al. The organization of the human cerebral cortex estimated by intrinsic

457 functional connectivity. Journal of neurophysiology (2011).

$45841 \quad$ Holmes, A. J. et al. Brain Genomics Superstruct Project initial data release with structural, functional, and behavioral measures. Scientific data 2, 1-16 (2015).

46042 Padmanabhan, J. L. et al. A Human Depression Circuit Derived From Focal Brain Lesions. Biol Psychiatry 86, 749-758, doi:10.1016/j.biopsych.2019.07.023 (2019).

43 Cotovio, G. et al. Mapping mania symptoms based on focal brain damage. J Clin Invest 130, 5209-5222, doi:10.1172/JCI136096 (2020). criminal behavior. Proc Natl Acad Sci U S A 115, 601-606,

do1:10.1073/pnas.1706587115 (2018).

45 Rossen, E., Kranzler, J. H. \& Algina, J. Confirmatory factor analysis of the MayerSalovey-Caruso emotional intelligence test V 2.0 (MSCEIT). Personality and individual Differences 44, 1258-1269 (2008).

46 Snider, S. B. et al. Cortical lesions causing loss of consciousness are anticorrelated with the dorsal brainstem. Hum Brain Mapp 41, 1520-1531, doi:10.1002/hbm.24892 (2020).

47 Bates, E. et al. Voxel-based lesion-symptom mapping. Nature neuroscience 6, 448-450 (2003).

48 Operskalski, J. T., Paul, E. J., Colom, R., Barbey, A. K. \& Grafman, J. H. Lesion mapping the four-factor structure of emotional intelligence. Frontiers in human neuroscience 9, 649 (2015).

49 Barbey, A. K., Colom, R. \& Grafman, J. Distributed neural system for emotional intelligence revealed by lesion mapping. Social cognitive and affective neuroscience $\mathbf{9}$, 265-272 (2014).

50 Gold, B. T. \& Buckner, R. L. Common prefrontal regions coactivate with dissociable posterior regions during controlled semantic and phonological tasks. Neuron 35, 803-812 (2002).

51 Aron, A. R., Robbins, T. W. \& Poldrack, R. A. Inhibition and the right inferior frontal cortex. Trends in cognitive sciences 8, 170-177 (2004).

52 Stuss, D. T. \& Alexander, M. P. Is there a dysexecutive syndrome? Philosophical Transactions of the Royal Society B: Biological Sciences 362, 901-915 (2007).

53 Gross, J. J. Antecedent-and response-focused emotion regulation: divergent consequences for experience, expression, and physiology. Journal of personality and social psychology 74, 224 (1998).

54 Gross, J. J. The emerging field of emotion regulation: An integrative review. Review of general psychology 2, 271-299 (1998).

55 He, Z., Liu, Z., Zhao, J., Elliott, R. \& Zhang, D. Improving emotion regulation of social exclusion in depression-prone individuals: a tDCS study targeting right VLPFC. Psychological medicine 50, 2768-2779 (2020).

56 Carrera, E. \& Tononi, G. Diaschisis: past, present, future. Brain 137, 2408-2422 (2014).

57 Adamaszek, M. et al. Consensus paper: cerebellum and emotion. The Cerebellum 16, 552-576 (2017). transcranial magnetic stimulation study. The Cerebellum 8, 28-34 (2009). 
59 Ferrucci, R. et al. Cerebellum and processing of negative facial emotions: cerebellar transcranial DC stimulation specifically enhances the emotional recognition of facial anger and sadness. Cognition \& emotion 26, 786-799 (2012).

60 Aldao, A., Gee, D. G., De Los Reyes, A. \& Seager, I. Emotion regulation as a transdiagnostic factor in the development of internalizing and externalizing psychopathology: Current and future directions. Development and psychopathology 28, 927-946 (2016).

61 Fernandez, K. C., Jazaieri, H. \& Gross, J. J. Emotion regulation: a transdiagnostic perspective on a new RDoC domain. Cognitive therapy and research 40, 426-440 (2016).

62 McGrogan, C. L., Dodd, A. L. \& Smith, M. A. Emotion regulation strategies in mania risk: a systematic review. Journal of clinical psychology 75, 2106-2118 (2019).

63 Sharma, N., Prakash, O., Sengar, K., Chaudhury, S. \& Singh, A. R. The relation between emotional intelligence and criminal behavior: A study among convicted criminals. Industrial psychiatry journal 24, 54 (2015).

64 Joormann, J. \& Gotlib, I. H. Emotion regulation in depression: Relation to cognitive inhibition. Cognition and Emotion 24, 281-298 (2010).

65 Berking, M., Wirtz, C. M., Svaldi, J. \& Hofmann, S. G. Emotion regulation predicts symptoms of depression over five years. Behaviour research and therapy 57, 13-20 (2014).

66 Megreya, A. M. \& Latzman, R. D. Individual differences in emotion regulation and face recognition. Plos one 15, e0243209 (2020).

67 Cohen, A. L. et al. Tuber Locations Associated with Infantile Spasms Map to a Common Brain Network. Annals of Neurology 89, 726-739 (2021). 


\section{Figures}

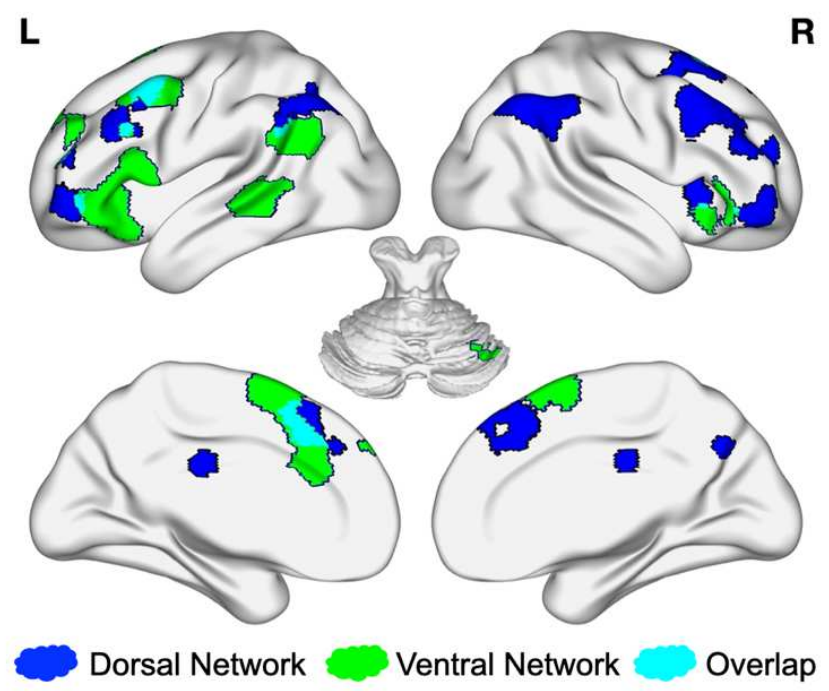

Figure 1. Meta-analysis identified two large-scale brain networks involved in emotion regulation tasks. A dorsal frontoparietal network ("Dorsal Network") and a ventral frontotemporal network ("Ventral Network") were identified in a recent clustering-based metaanalysis of existing whole-brain fMRI studies on emotion regulation (figure adapted from Morawets et al., 2020). 
A. Overlap of lesion locations across patients
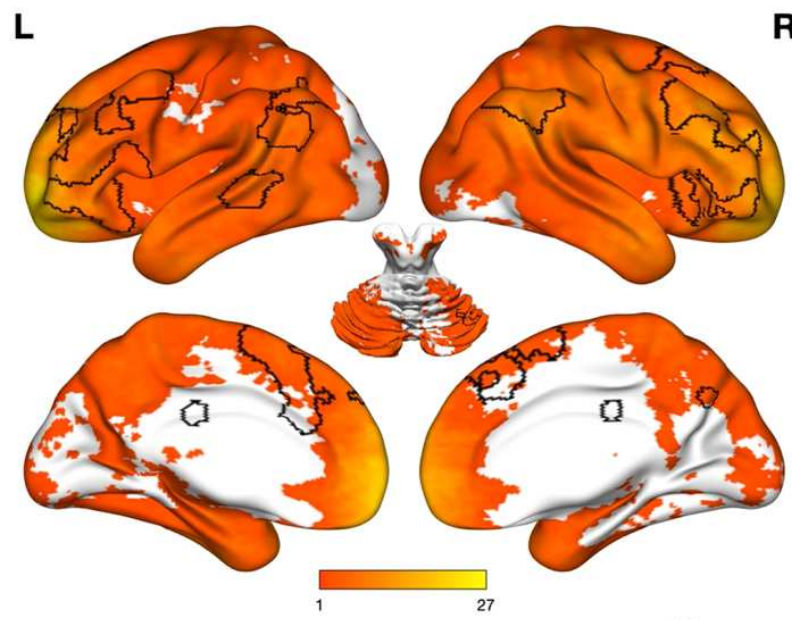

\# of patients with lesions
B. Examples of lesion locations in 4 patients

$\mathbf{R}$

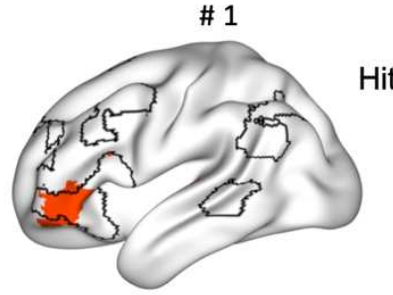

\# 3

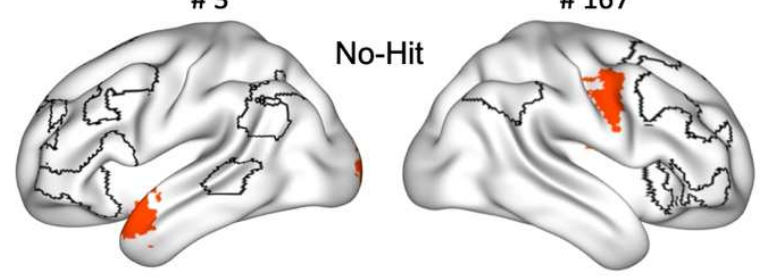

Lesion voxels
Figure 2. Lesion locations and intersections with the overall a priori networks. A. Overlap of lesion locations across all patients $(n=167)$. The red-yellow color indicates the range in the number of patients with lesions at a given voxel (maximum $=27)$. a priori brain networks for emotion regulation are shown using a black outline. B. Examples of lesion locations intersecting with a priori networks (Hit group) versus not (No-Hit group). 

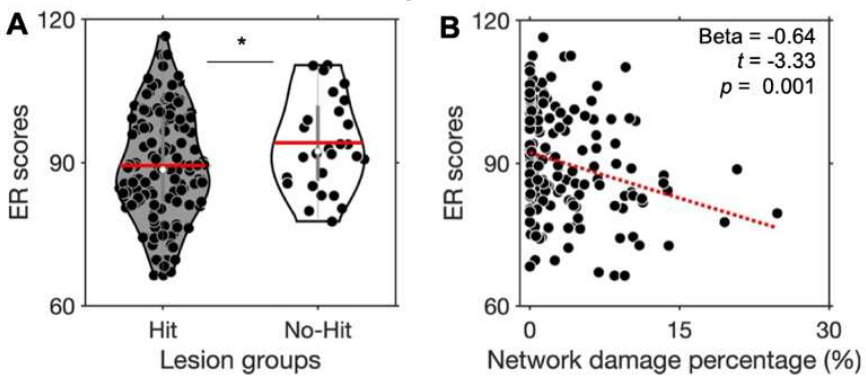

Dorsal Network
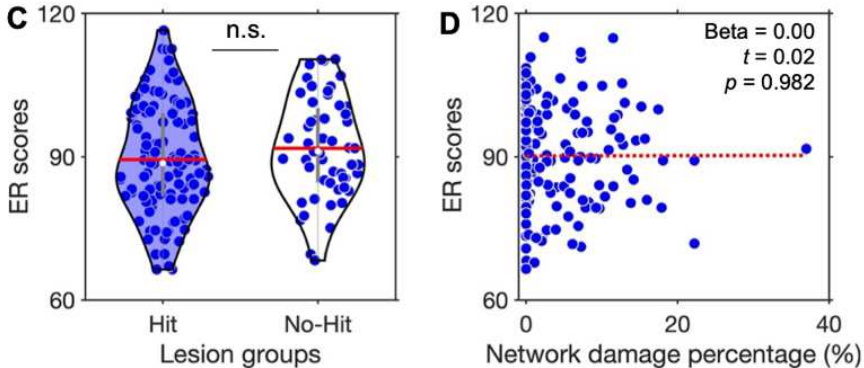

Ventral Network
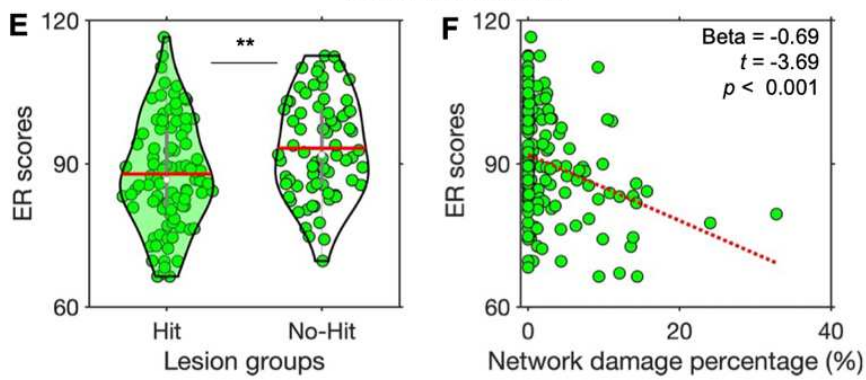

Figure 3. Damage to the ventral but not the dorsal a priori network is associated with emotion regulation impairment. A-B. Emotion regulation scores were significantly lower for patients with lesions intersecting the overall a priori networks, i.e., Hit vs No-Hit, and were significantly associated with network damage percentage on the overall a priori networks. C-D. Emotion regulation scores did not differ significantly between the two lesion groups on the Dorsal Network and were not significantly associated with network damage percentage on the Dorsal Network. E-F. Emotion regulation scores were significantly lower for patients with lesions intersecting the Ventral Network, i.e., Hit vs No-Hit, and were significantly associated with network damage percentage on Ventral Network. ER, emotion regulation. 

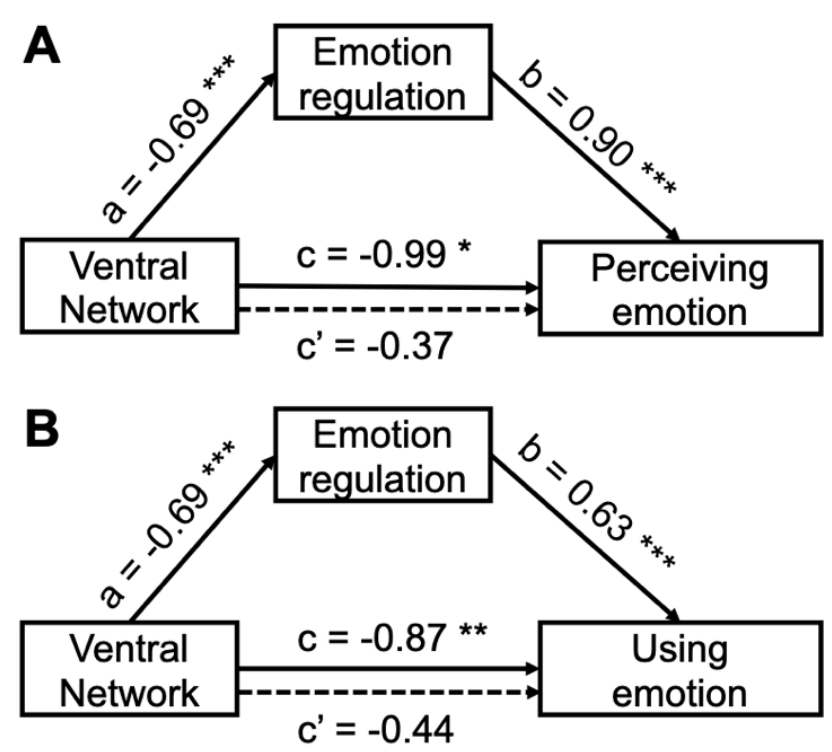

\section{Figure 4. Emotion regulation fully mediated the effect of damage to the Ventral Network} on other emotion processing abilities. c, regression coefficient for the total effect of an independent variable (IV: network damage percentage of Ventral Network) on a dependent variable (DV: Perceiving/Using/Understanding emotion scores) (Model I). a, regression coefficient for effect of the IV on a mediator (M: Emotion regulation) (Model II). b, regression coefficient for effect of the M on the DV when $\mathrm{M}$ and IV are both included as IVs in a regression model (Model III). c', regression coefficient for the direct effect of the IV on the DV in Model

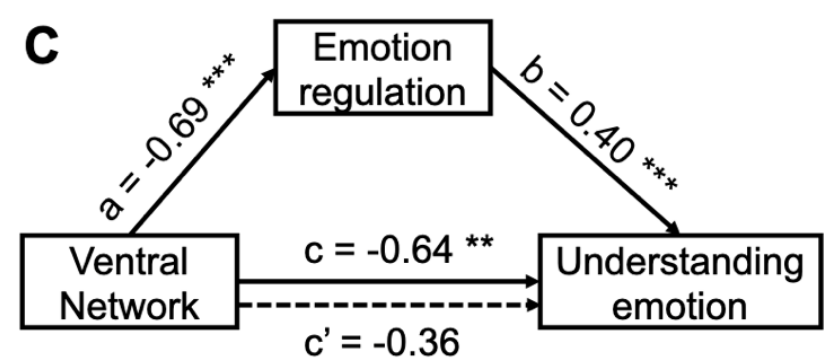
III. $*: p<0.05 ; * *: p<0.01 ; * * *: p<0.001$ 
A. Lesion locations
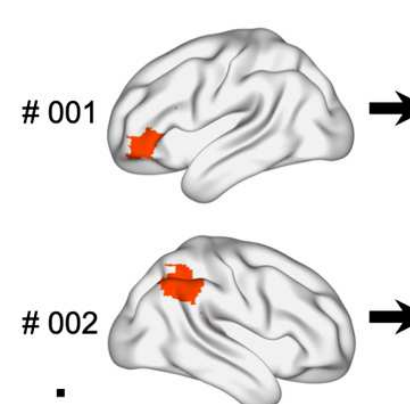

:

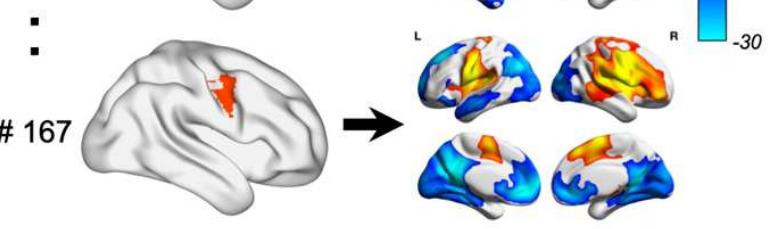

C. Lesion connectivity associated with emotion regulation scores

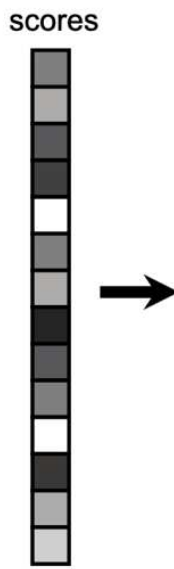

$\mathbf{L}$
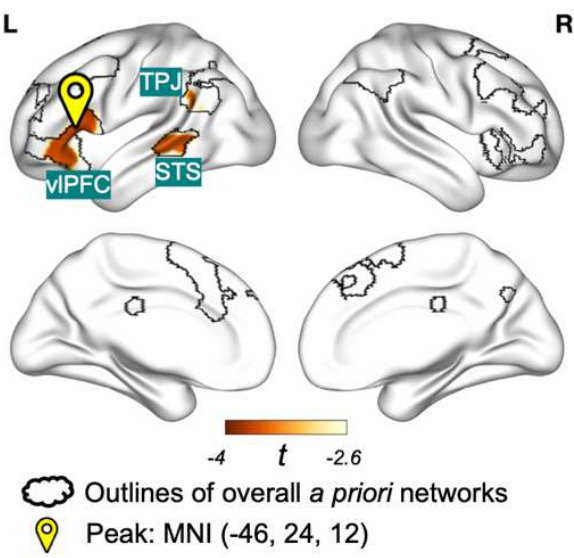

562

563

564

565

566

567

568

569

570

Figure 5. Lesion connectivity to the left vIPFC is associated with emotion regulation impairment. A. Lesion locations from three representative patients. B. Lesion connectivity maps for each patient, i.e., RSFC maps using individual lesions as seed regions combined with a normative connectome, shown here thresholded at $t= \pm 10$. C. Comparing these maps to emotion regulation scores from each patient identified critical negative correlations with several regions including the left vlPFC, STS, and TPJ (voxel-wise $F D R p<0.05, k>10$ ). vlPFC, ventrolateral prefrontal cortex; STS, superior temporal sulcus; TPJ, temporoparietal junction. 
A. vIPFC-seeded FC map

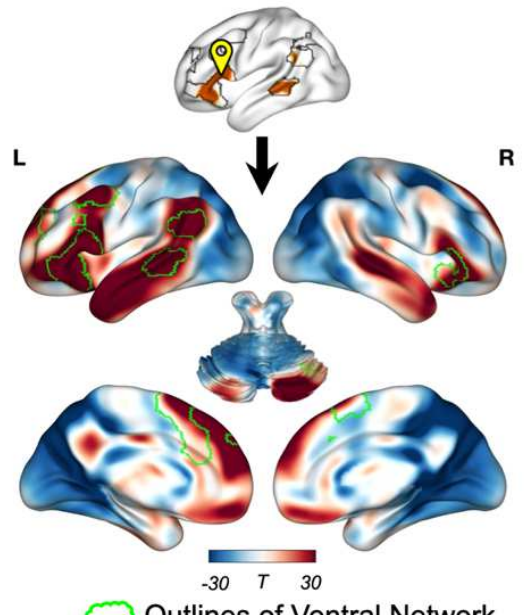

B. Network damage score computation

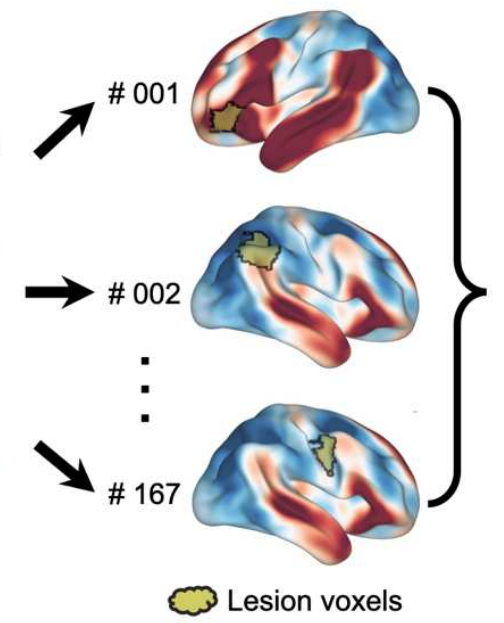

C. Network damage scores are associated with emotion regulation scores

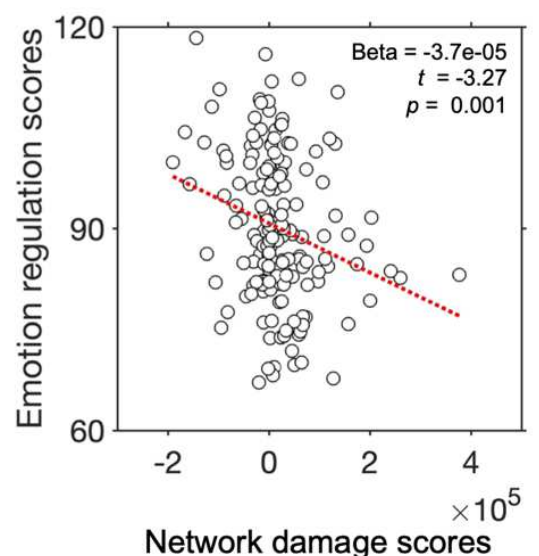

Figure 6. Network damage scores on a vIPFC-seeded FC map are associated with emotion regulation impairment. A. Lesion network mapping suggests that FC with the vlPFC defines a potential brain network important for impaired emotion regulation. Darker red/blue represents stronger positive/negative FC with the left vlPFC cluster. B. Network damage scores can be computed based on the sum of FC $(T)$ values at lesion voxels within the vlPFC-seeded map. C. As confirmation, network damage scores (controlled for lesion size) on the vlPFC-seeded FC map showed a significant negative correlation with emotion regulation scores using our original data. FC, functional connectivity. 


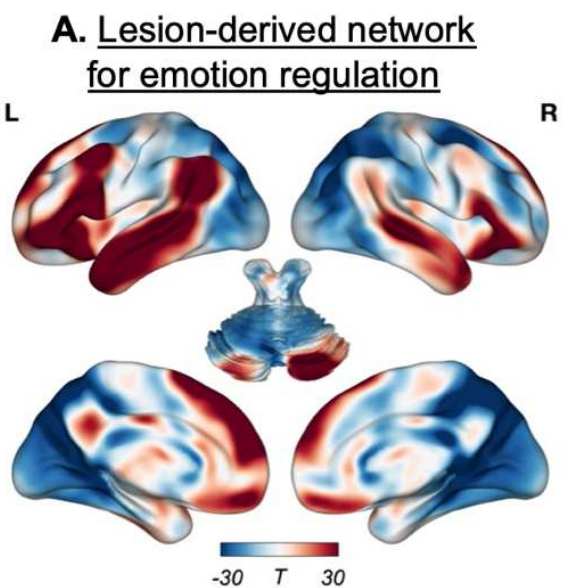

B. Network damage scores of disorder-causing lesions

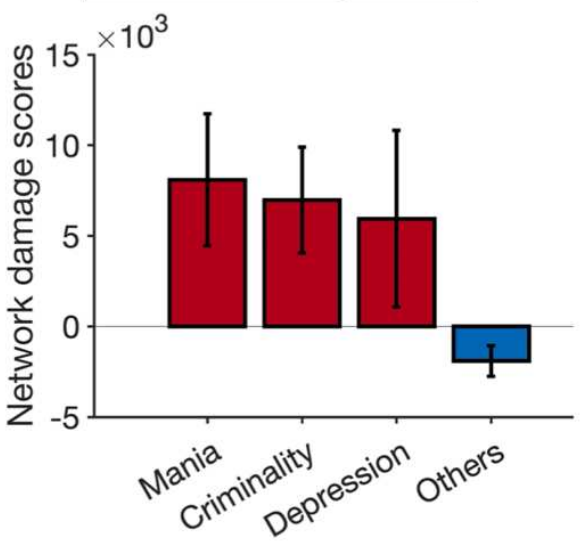

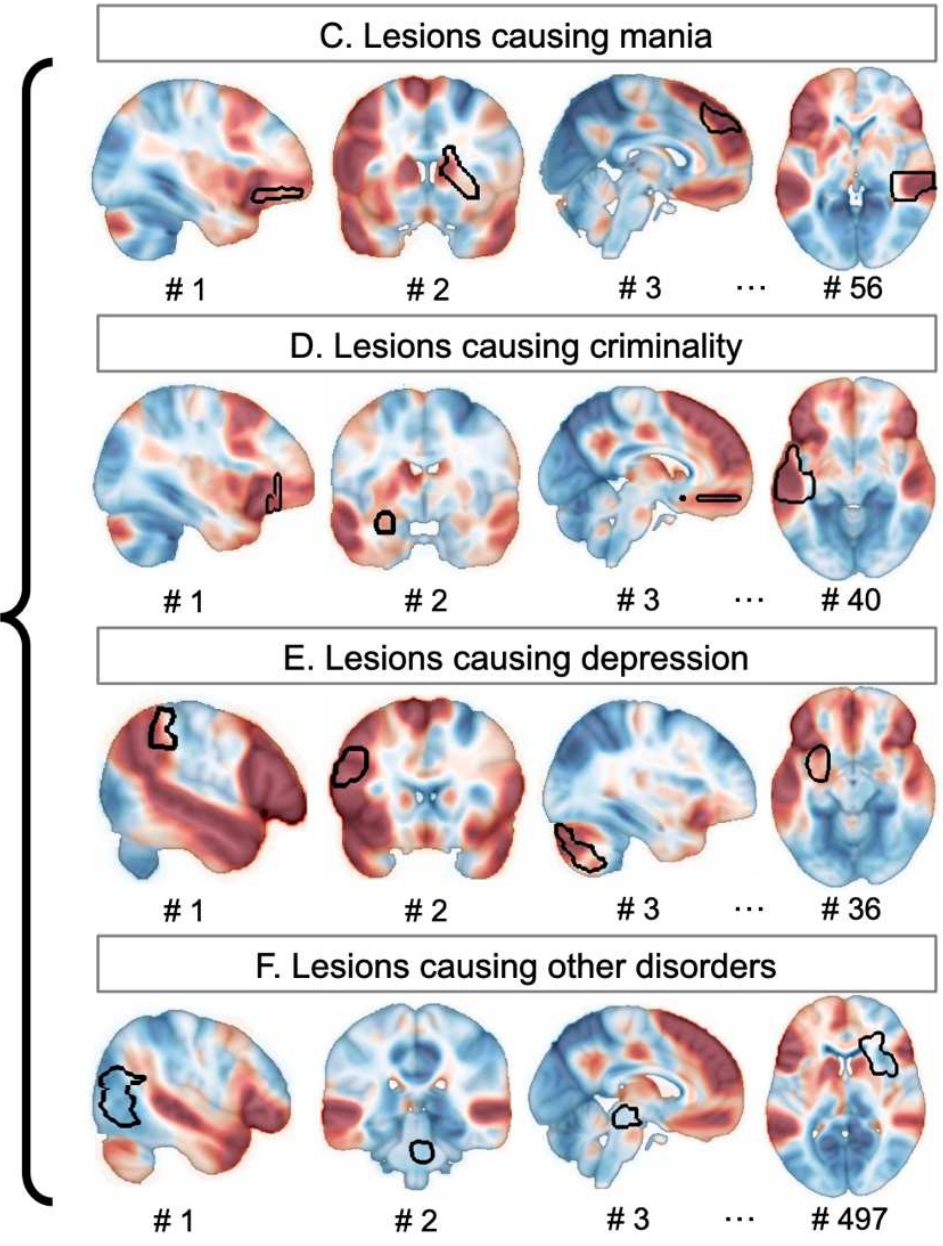

Figure 7. Relations between lesions intersecting with lesion-derived emotion regulation network and diverse disorders. A. The left vlPFC-seeded FC map defines a lesion-derived network for emotion regulation. B. Network damage scores, i.e., the sum of FC values (controlled for lesion size) of the lesion-derived emotion regulation network at lesion locations is elevated for disorders associated with impaired emotion regulation. Error bars represent standard error across lesions within each disorder. C-F. Representative lesion locations (black outlines) causing mania, criminality, and depression more often intersect positive areas of our lesionderived network for emotion regulation than other disorders. 


\section{Supplementary Information}

\section{Title: A lesion-derived brain network for emotion regulation}

\section{Authors:}

Jing Jiang ${ }^{1,2, *}$, Michael A. Ferguson ${ }^{1,2}$, Jordan Grafman ${ }^{4,5}$, Alexander L. Cohen ${ }^{1,2,3, \#}$ and Michael D. Fox ${ }^{1,2, \#}$

601 6. Center for Brain Circuit Therapeutics, Brigham \& Women's Hospital, Boston, MA, USA

602 7. Department of Neurology, Harvard Medical School, Boston, MA, USA

603 8. Department of Neurology, Boston Children's Hospital, Boston, MA, USA

604 9. Feinberg School of Medicine, Northwestern University, Chicago, IL, USA

605 10. Shirley Ryan Ability Lab, Chicago, IL, USA

606

607

608

609

610

* Corresponding author

611 Jing Jiang, Ph.D.

Center for Brain Circuit Therapeutics,

75 Francis St, Boston, MA 02115

612 jiiang17@,bwh.harvard.edu

613

614 \# Contributed equally to this work

615

Key Words

617

618

619

Emotion regulation, functional connectivity, brain lesion, lesion network mapping, fMRI performed data analyses. J.J wrote manuscript drafts. J.G. provided lesions with behavioral and demographic data. All authors revised manuscript. 
A. \# of patients with lesions hitting a priori networks

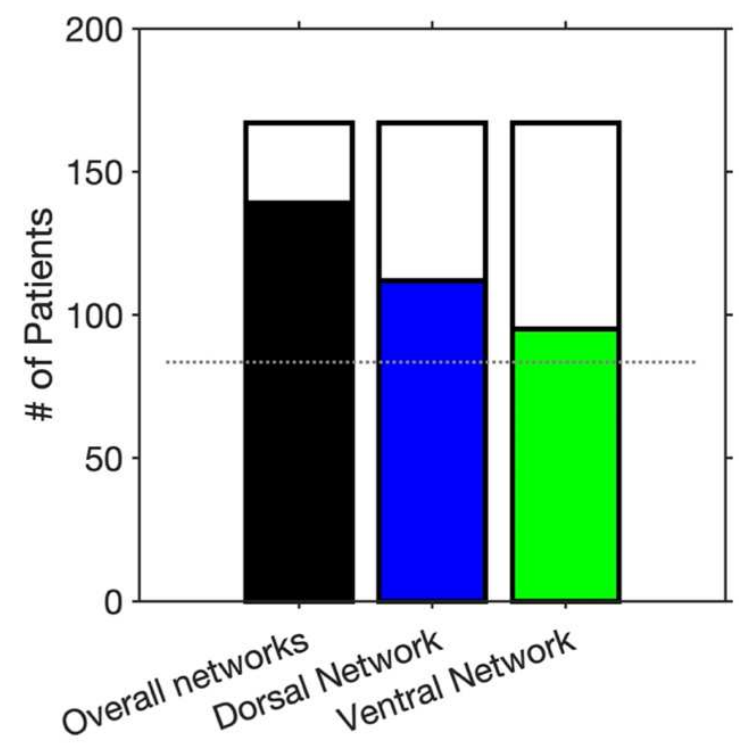

B. Distribution of individual network damage percentages

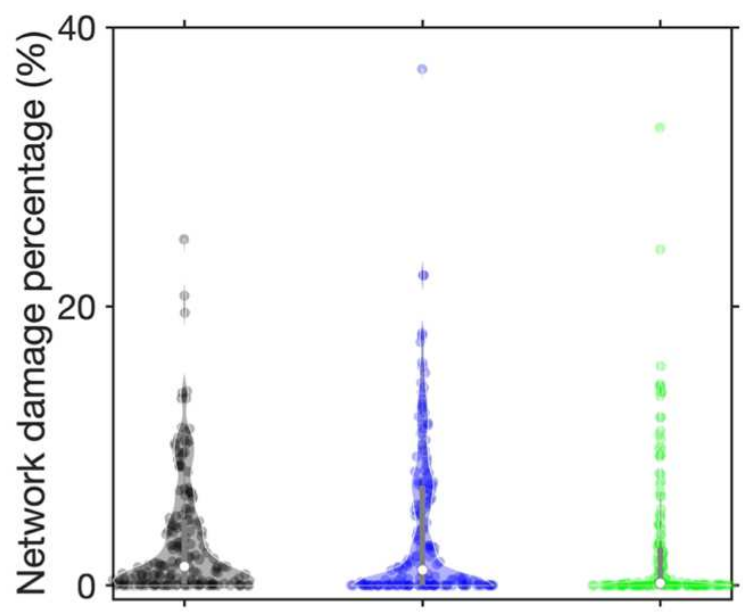

624

625

626
Fig. S1. Number of patients hitting a priori ER networks (A) and distribution of individual network damage percentages (B). 


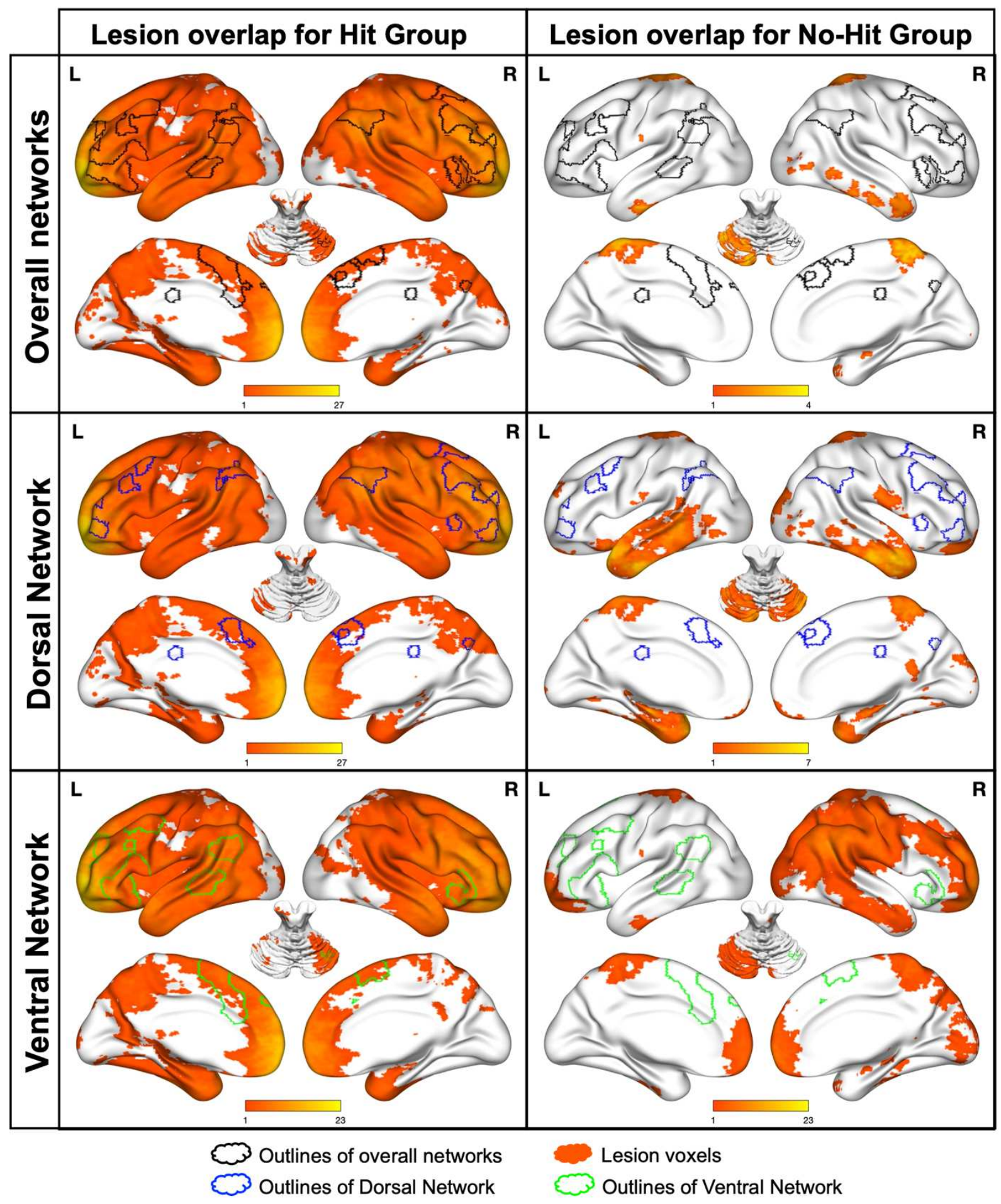

Fig. S2. Overlaps of lesion locations across patients in Hit and No-Hit group on the a priori

629 networks. Red-yellow color indicates the range in the number of patients with lesions at a given 630 voxel. 

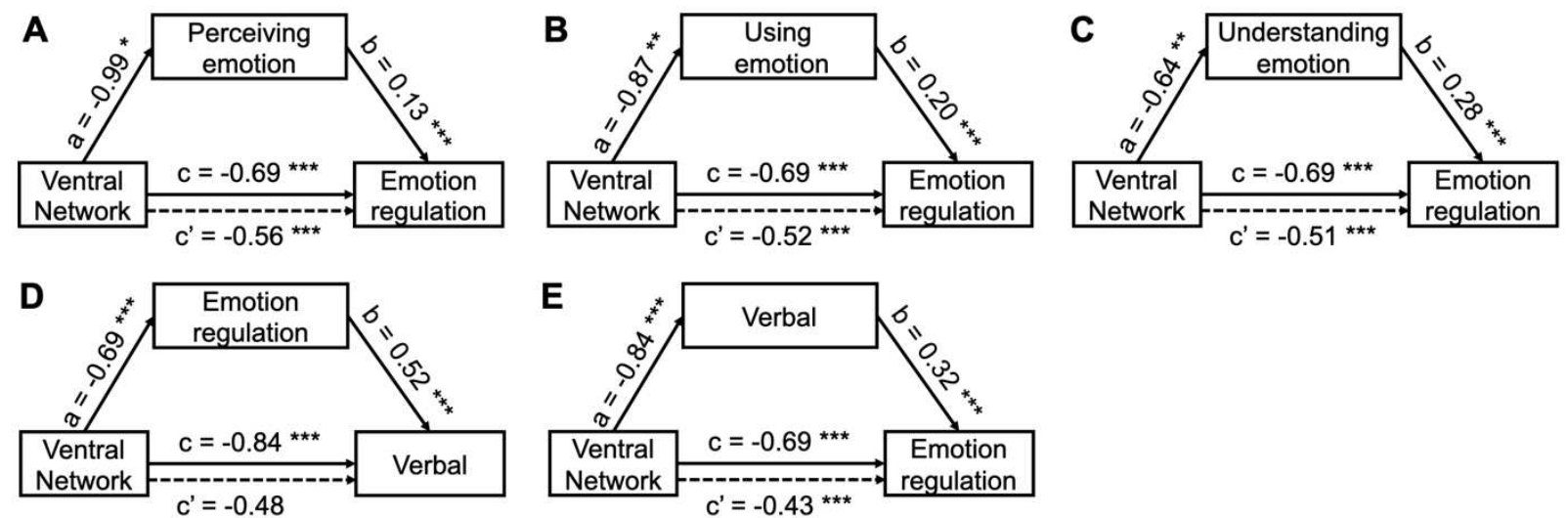

Fig. S3. Mediation analyses revealed the specificity of Ventral Network in emotion

633 regulation. A-C. Other emotion processing abilities ONLY partially mediated the effect of

634 damage to the Ventral Network on ER ability. D. Emotion regulation fully mediated the effect of

635 damage to the Ventral Network on Verbal abilities. E. Verbal ability ability ONLY partially mediated the effect of damage to the Ventral Network on ER ability. c, regression coefficient for the total effect of an independent variable (IV) on a dependent variable (DV) (Model I). a,

638 regression coefficient for effect of the IV on a mediator (M) (Model II). b, regression coefficient for effect of the $\mathrm{M}$ on the DV when $\mathrm{M}$ and IV are both included as independent predictors in a regression model (Model III). c', regression coefficient for the direct effect of the IV on the DV in Model III. *: $p<0.05 ; * *: p<0.01 ; * * *: p<0.001$ 

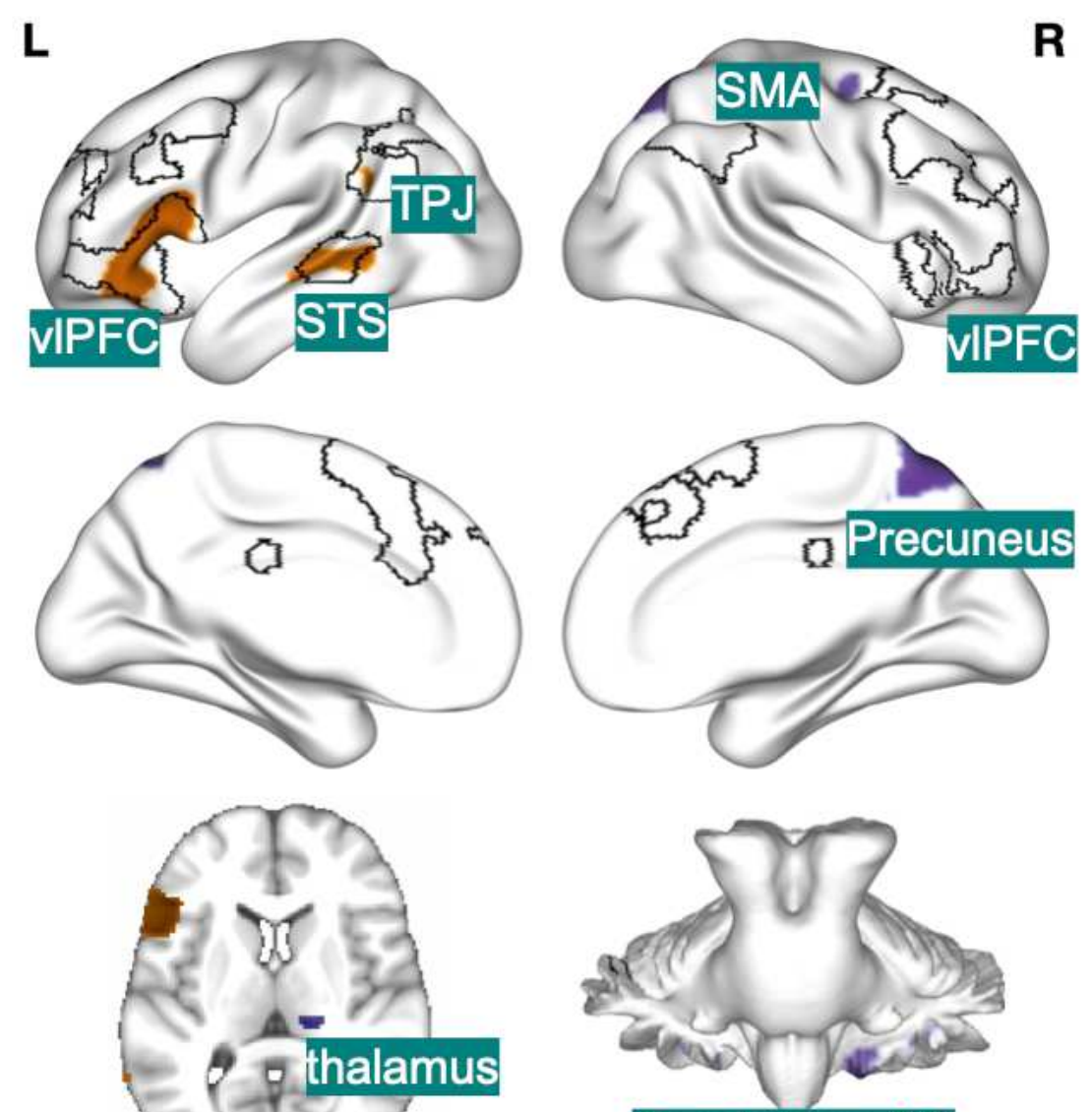

Cerebellum 8/9

$t$
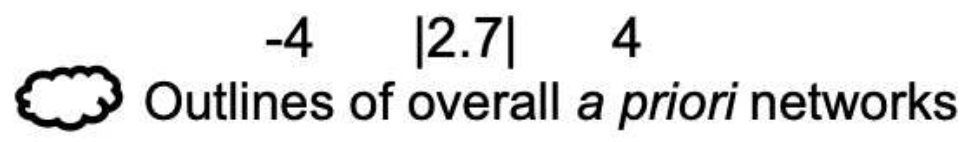

Fig S4. Lesion connectivity predicts ER at the whole-brain level. Lesion connectivity with regions including the left vlPFC, STS, TPJ, and right vlPFC showed significant negative correlation with ER scores, while that including Precuneus, bilateral Cerebellum, right thalamus, and right SMA showed significant positive correlation with ER scores (whole-brain voxel-wise $p$ $<0.005, k>30$ ). vlPFC, ventrolateral prefrontal cortex; STS, superior temporal cortex; TPJ, temporoparietal junction; SMA, supplementary motor area. 
A. vIPFC-seeded FC map excluding Ventral Network

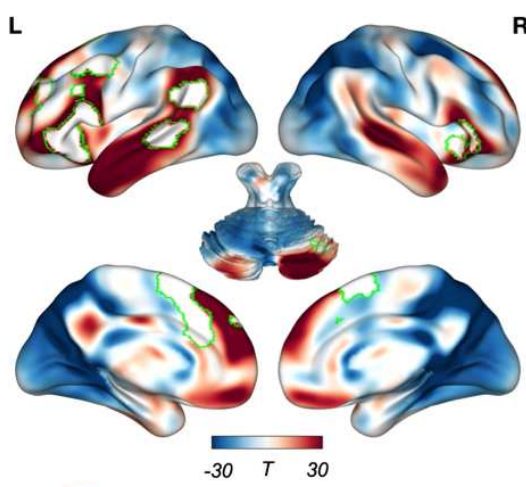

Outlines of Ventral Network
B. Network damage score computation

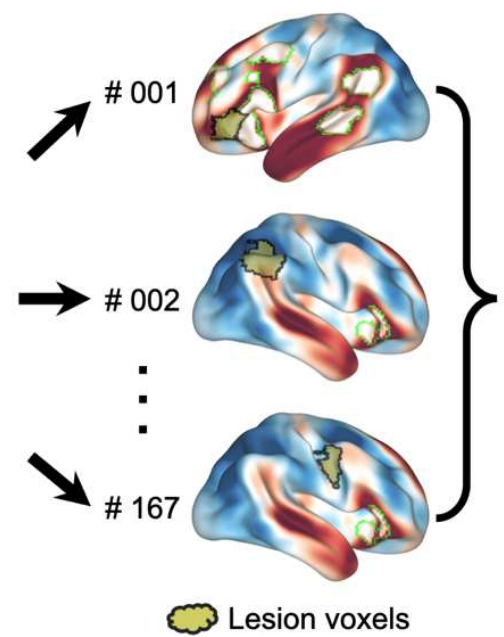

C. Network damage scores predict emotion regulation scores

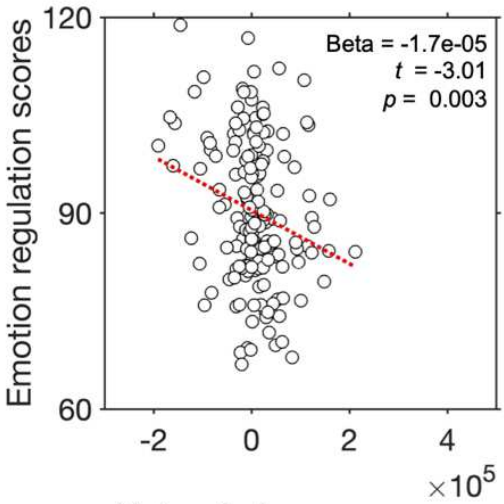

Network damage scores
653

654

655

656

657

658

659

660

661

662
Fig. S5. Network damage scores on the vIPFC-seeded FC map excluding Ventral Network are associated with emotion regulation impairment. A. vlPFC-seeded FC map excluding Ventral Network. B. Network damage scores can be computed based on the sum of FC $(T)$ values at lesion voxels within the vlPFC-seeded map excluding Ventral Network. C. As confirmation, network damage scores (controlled for lesion size) on the vlPFC-seeded FC map excluding Ventral Network showed a significant negative correlation with emotion regulation scores using our original data. 

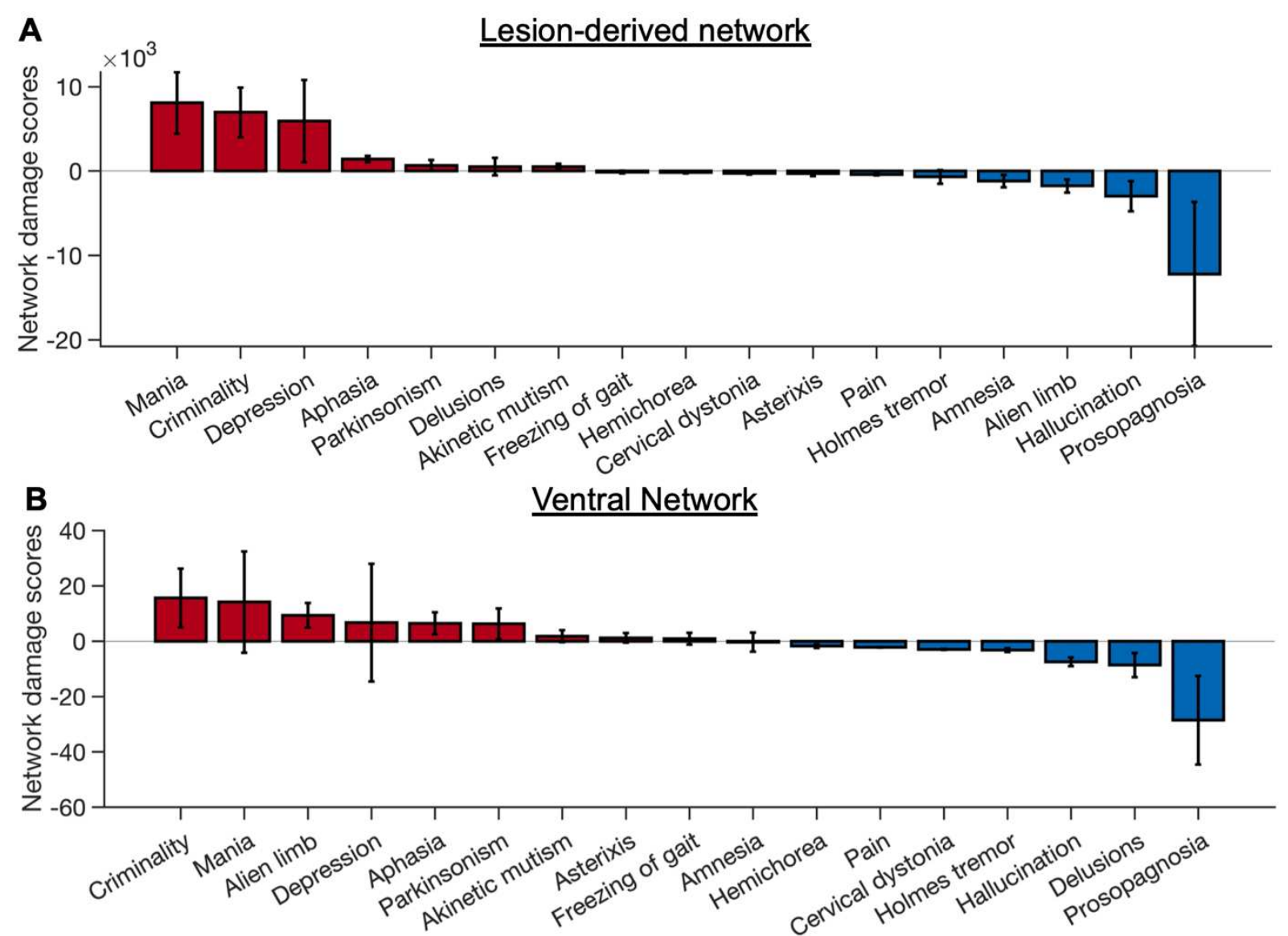

663

664

665

666

667

668

669

670
Fig. S6. Network damage scores of lesions causing a variety of disorders. A. Network damage scores based on sum of FC values (controlled for lesion size) in the intersection of lesions with our lesion-derived network for emotion regulation. B. Network damage scores based on sum of voxels (controlled for lesion size) in the intersection of lesions with the Ventral Network for emotion regulation. Error bars represent standard error across lesions within each disorder. 